\title{
A new interpretation of dielectric data in molecular glass formers
}

\author{
U. Buchenau, ${ }^{a)}$ M. Ohl, and A. Wischnewski \\ Institut für Festkörperforschung, Forschungszentrum Jülich, Postfach 1913, D-52425 Jülich, \\ Federal Republic of Germany
}

(Received 17 November 2005; accepted 17 January 2006; published online 7 March 2006)

\begin{abstract}
Literature dielectric data of glycerol, propylene carbonate, and ortho-terphenyl show that the measured dielectric relaxation is a decade faster than the Debye expectation but still a decade slower than the breakdown of the shear modulus. From a comparison of time scales, the dielectric relaxation seems to be due to a process which relaxes not only the molecular orientation but also the entropy, the short range order, and the density. On the basis of this finding, we propose an alternative to the Gemant-DiMarzio-Bishop extension of the Debye picture. (C) 2006 American Institute of Physics. [DOI: 10.1063/1.2176618]
\end{abstract}

Broadband dielectric spectroscopy has developed into the most important tool for the study of glass formers. It is able to cover the whole relevant frequency range, from microhertz to terahertz. ${ }^{1}$ Therefore it would be very desirable to understand the dielectric susceptibility in terms of physical processes. In particular, one would like to link the $\alpha$ peak of the dielectric data to the disappearance of the shear modulus at long times, the essence of the flow process.

Such a link is in principle provided by the GemantDiMarzio-Bishop $^{2,3}$ (GDB) extension of Debye's treatment. The extension considers the molecule as a small sphere with a hydrodynamic radius $r_{H}$ immersed in the viscoelastic liquid. The medium is characterized by a frequency-dependent complex shear modulus

$$
G(\omega)=G_{\infty} g(\omega),
$$

where $G_{\infty}$ is the infinite frequency shear modulus and $g(\omega)$ is a normalized complex function, increasing from zero to 1 as the frequency goes from zero to infinity.

For molecules with a weak dipole moment such as ortho-terphenyl (OTP), the GDB extension is

$$
\frac{\epsilon(\omega)-n^{2}}{\epsilon_{\mathrm{low}}-n^{2}}=\frac{1}{1+c_{r} g(\omega)},
$$

with

$$
c_{r}=\frac{4 \pi G_{\infty} r_{H}^{3}}{k_{B} T} .
$$

Here $\epsilon(\omega)$ is the complex frequency-dependent dielectric constant (with the conductivity contribution already subtracted), $n$ is the refractive index, $\epsilon_{\text {low }}$ is the low-frequency limit of $\epsilon$, and $T$ is the temperature. One needs only the knowledge of the molecular radius $r_{H}$. Then one can calculate $\epsilon$ from measurable quantities.

For strongly polar molecules such as glycerol and propylene carbonate, one should take the difference between the external applied electric field and the internal field seen by

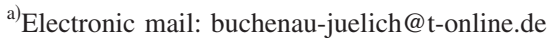

the molecule into account. ${ }^{4}$ In Onsager's scheme, ${ }^{5}$ extended to the dynamics by Fatuzzo and Mason ${ }^{6}$ and reformulated by Niss and Jakobsen,

$$
\frac{\epsilon(\omega)-n^{2}}{\epsilon_{\mathrm{low}}-n^{2}} \frac{2 \epsilon_{\mathrm{low}} \epsilon(\omega)+\epsilon_{\mathrm{low}} n^{2}}{\epsilon^{2}(\omega)+\epsilon(\omega) \epsilon_{\mathrm{low}}+\epsilon_{\mathrm{low}} n^{2}}=\frac{1}{1+c_{r} g(\omega)} .
$$

This complex quadratic equation still allows to calculate $\epsilon(\omega)$ from $G(\omega)$, provided that $r_{H}$ is known.

The molecular radius can be determined from NMR field gradient diffusion data for glycerol ${ }^{8}\left(r_{H}=0.16 \mathrm{~nm}\right)$ and for propylene carbonate ${ }^{9}\left(r_{H}=0.26 \mathrm{~nm}\right)$ via the Stokes-Einstein equation. For glycerol, there is a dynamic shear modulus measurement ${ }^{10}$ in the temperature range close to the glass transition. Using the shift factors of this measurement, one can calculate $G(\omega)$ at the temperature of $196 \mathrm{~K}$ of a dielectric measurement. ${ }^{11}$ At this temperature, ${ }^{12} n^{2}=2.26$. Figure 1(a) compares the GDB expectation of Eq. (4) with these values

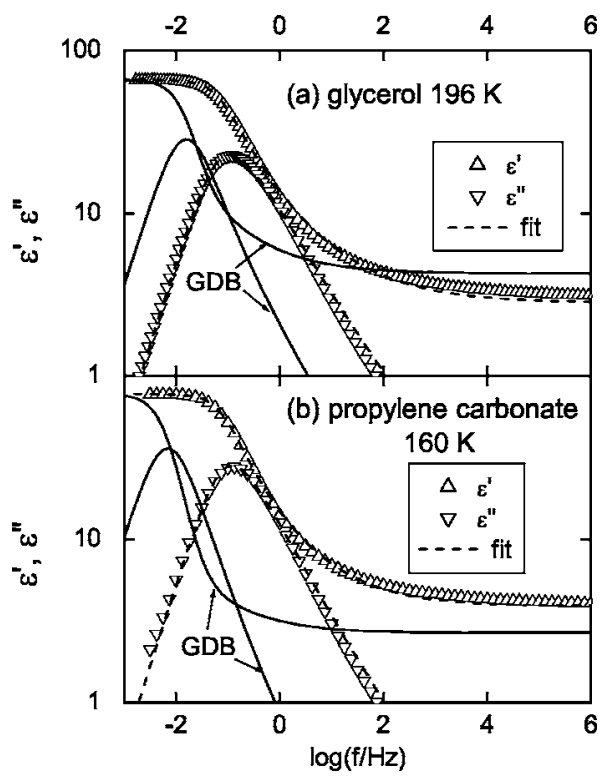

FIG. 1. Gemant-DiMarzio-Bishop (GDB) expectation (continuous lines) compared to (a) dielectric data (Ref. 11) of glycerol at $196 \mathrm{~K}$ (b) dielectric data (Ref. 15) of propylene carbonate at $160 \mathrm{~K}$. The better fits (dashed lines) are explained in the text. 
to the measured data. The calculation underestimates the peak frequency in $\epsilon^{\prime \prime}$ by an order of magnitude, a discrepancy which has been noted earlier. ${ }^{8}$

The same holds for propylene carbonate. There is a shear modulus measurement ${ }^{13}$ at $159 \mathrm{~K}$, close to the glass transition. Figure 1(b) compares the GDB prediction (with $n^{2}$ $=2.19)^{14}$ to dielectric data ${ }^{15}$ at $160 \mathrm{~K}$. Again, we find the peak in $\epsilon^{\prime \prime}$ shifted by a decade. Obviously, the undercooled liquid finds a much faster way to relax the molecular orientation than the Debye mechanism of a molecular sphere rotating in a viscous liquid.

As pointed out by Niss et al., ${ }^{16}$ one does not even get a good fit if one adapts the molecular radius, because if one adapts the peak in $\epsilon^{\prime \prime}$, the high-frequency $\epsilon^{\prime}$ gets much too high.

In order to find out which mechanism might be responsible for the decay of the molecular orientation, we compare the dielectric relaxation times to the ones determined by other techniques, in the spirit of earlier comparisons by Ngai and Rendell, ${ }^{17}$ Blochowicz et al., ${ }^{18}$ and Schröter and Donth. ${ }^{13}$ We recalculate all data in terms of a KohlrauschWilliams-Watts $(\mathrm{KWW})$ decay in time $\exp \left(-\left(t / \tau_{\mathrm{KWW}}\right)^{\beta}\right)$, either by using pragmatical recipes from the literature ${ }^{19,20}$ or by refitting the data. To get rid of the strong temperature dependence of the viscosity, the resulting KWW relaxation time is divided by the Maxwell time $\tau_{\text {Maxwell }}=\eta / G_{\infty}$.

The choice of a Kohlrausch or KWW function is practical for the following reasons: (i) it very often gives a good fit; (ii) the inverse of the absorption peak frequency in $\omega$ is close to $\tau_{\mathrm{KWW}}$, so one compares peak frequencies, independent of the stretching parameter $\beta$; and (iii) for a shear modulus following the Kohlrausch function,

$$
\frac{\tau_{\mathrm{KWW}}}{\tau_{\text {Maxwell }}}=\frac{\beta}{\Gamma(1 / \beta)} .
$$

Usually, $\beta$ lies between 0.4 and 0.6 , so the ratio should be between one-third and two-thirds; the Kohlrausch relaxation time should be a factor of 1.5-3 shorter than the Maxwell time. (iv) For a shear compliance measurement such as the one $^{21}$ in OTP, the steady-state compliance $J_{e}^{0}$ obeys the relation $^{22}$

$$
J_{e}^{0} \eta^{2}=\int_{0}^{\infty} t G(t) d t
$$

so for a Kohlrausch function ${ }^{20}$

$$
J_{e}^{0} G_{\infty}=\frac{\Gamma(2 / \beta) \beta}{\Gamma(1 / \beta)} .
$$

Since the compliance measurement supplies all three values $J_{e}^{0}, \eta$, and $G_{\infty}$, one can determine $\beta$ and $\tau_{\mathrm{KWw}} / \tau_{\text {Maxwell }}$ without calculating $\tau_{\text {Maxwell }}$.

As usual, the measured viscosity $\eta$ of our three substances is fitted in terms of a combination of two VogelFulcher-Tammann-Hesse laws,
TABLE I. Viscosity and shear modulus parameters. For references see text.

\begin{tabular}{lccc}
\hline \hline Substance & Glycerol & Propylene carbonate & OTP \\
\hline $\log \left(\eta_{01}\right)(\mathrm{Pa} \mathrm{s})$ & -7.1 & -8.92 & -11.89 \\
$B_{1}(\mathrm{~K})$ & 1260 & 667 & 1461.2 \\
$T_{01}(\mathrm{~K})$ & 118 & 122 & 178.4 \\
$T_{1}(\mathrm{~K})$ & 283 & 193 & 310 \\
$\log \left(\eta_{02}\right)(\mathrm{Pa} \mathrm{s})$ & -5.45 & -3.91 & -4.24 \\
$B_{2}(\mathrm{~K})$ & 780 & 191 & 245.9 \\
$T_{02}(\mathrm{~K})$ & 153 & 150 & 241.72 \\
$T_{2}(\mathrm{~K})$ & 283 & 175 & 275 \\
$T_{g}(\mathrm{~K})$ & 187 & 157 & 243 \\
$G_{\infty}\left(T_{g}\right)(\mathrm{GPa})$ & 4.58 & 2.5 & 1.6 \\
$a\left(\mathrm{~K}^{-1}\right)$ & 0.023 & 0.007 & 0.0057 \\
$b\left(\mathrm{~K}^{-2}\right)$ & $2.1 \times 10^{-5}$ & $2 \times 10^{-5}$ & $1.4 \times 10^{-5}$ \\
\hline \hline
\end{tabular}

$\log \eta=\log \eta_{0 i}+\frac{B_{i}}{T-T_{0 i}}$,

with $i=1$ and $i=2$, respectively. The first of these two is valid below a temperature $T_{1}$, the second above a temperature $T_{2}$ $\leqslant T_{1}$. Between $T_{2}$ and $T_{1}$, one takes a linear interpolation between the two to ensure continuity.

The infinity frequency shear modulus $G_{\infty}$ is parametrized in terms of a Taylor expansion around the glass temperature $T_{g}$,

$$
G_{\infty}=G_{\infty}\left(T_{g}\right)\left(1-a\left(T-T_{g}\right)+b\left(T-T_{g}\right)^{2}\right) .
$$

The parameters of these two equations for the viscosity and the infinite frequency shear modulus are listed in Table I. For glycerol $^{10}$ and propylene carbonate, ${ }^{23}$ the Vogel-Fulcher parameters were taken from viscosity data fits in the literature. For OTP, we fitted our own parameters to the many viscosity measurements in the literature..$^{21,24-27}$ In glycerol, $G_{\infty}$ was fitted to the Brillouin shear wave measurement of Scarponi et al. ${ }^{28}$ For propylene carbonate, there is no Brillouin shear wave measurement. Therefore, the infinite frequency shear modulus had to be taken from a longitudinal Brillouin measurement, ${ }^{29}$ assuming $G_{\infty}=c_{11} / 4$. In OTP, there is a shear wave Brillouin scattering measurement, ${ }^{30}$ but the $G_{\infty}$ values from this measurement extrapolate to zero already at $308 \mathrm{~K}$. Therefore we took $G_{\infty}\left(T_{g}\right)$ from this measurement but determined the parameters $a$ and $b$ of Eq. (9) from the combined evaluation of light and x-ray Brillouin scatterings of Monaco et al., ${ }^{31}$ assuming the same temperature dependence for the infinite frequency longitudinal and shear moduli.

We start the comparison for glycerol in Fig. 2. The normalized dielectric ${ }^{1,11,32} \mathrm{KWW}$ relaxation times are compared to those from mechanical data, ${ }^{10,12,13,33-37}$ from dynamic heat capacity measurements, ${ }^{38,39}$ from NMR, ${ }^{40}$ from photon correlation spectroscopy (PCS), ${ }^{41}$ from transient grating (TG), ${ }^{37}$ and from neutron spin-echo measurements at the first sharp diffraction peak. ${ }^{42}$ Dynamic light scattering (DLS) data ${ }^{43}$ (not shown in Fig. 2) tend to lie between dielectrics and mechanics, but otherwise the figure corroborates the earlier conclusion of Schröter and Donth, ${ }^{13}$ namely, that there seems to be a grouping into the faster mechanical relaxation and a slower heat capacity, dielectric, NMR, PCS, TG, and neutron 


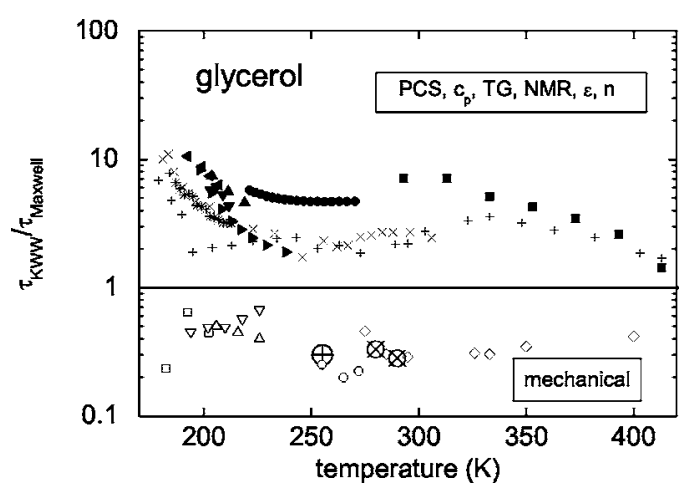

FIG. 2. Kohlrausch-Williams-Watts relaxation times in glycerol, normalized to the Maxwell time as described in the text. Symbols: open squares: shear (Ref. 13), open up triangles: shear (Ref. 34), open down triangles: compression (Ref. 35), open circles: longitudinal acoustic (Ref. 36), open circle with plus: shear and compression (Ref. 33), open circles with cross: TG (Ref. 37); open diamonds: longitudinal Brillouin (Ref. 12), pluses: dielectric (Ref. 1), crosses: dielectric (Ref. 11), asterisks: dielectric (Ref. 32), full up triangles: heat capacity (Ref. 38), full down triangles: heat capacity (Ref. 39), full left triangles: NMR (Ref. 40), full right triangles: PCS (Ref. 41), full circles: TG (Ref. 37), and full squares: neutron spin echo (Ref. 42).

spin-echo relaxation. The mechanical relaxation times follow the expectation of Eq. (5) within reasonable error limits. The others tend to lie a factor of about ten higher. With changing temperature, both time scales move together with a roughly constant separation. This shows that the misfit of the Debye result is temperature independent, unlike the deviations from the Stokes-Einstein relation at lower temperatures. ${ }^{8}$ We will come back to this point in the discussion of OTP. Here, let us first discuss what one sees in each technique.

In the case of mechanical and dielectric data, there can be large differences in relaxation time between a modulus and the corresponding compliance. This difference is negligible if the relative change of the quantity in question is small, but here we deal with large relative changes. Therefore one has to check whether mechanical moduli and dielectric susceptibility are the correct choices.

For the mechanical data, there is a good physical reason to choose the moduli rather than the compliances, because this choice leads to practically the same relaxation time for the bulk and the shear modulus. ${ }^{33,44,45}$ In fact, glycerol was one of the first cases in which this equality was demonstrated by the longitudinal and shear ultrasonic data of Piccirelli and Litovitz $^{33}$ (the circle with a plus in Fig. 2 at $255 \mathrm{~K}$ ). In Fig. 2 , it is again demonstrated at lower frequencies by the good agreement between the shear measurements ${ }^{10,13,34}$ and the compression measurement of Christensen and Olsen. ${ }^{35}$ If one goes over to compliances, this good agreement gets lost. ${ }^{46}$

Similarly, in the dielectric case one should take the dielectric constant rather than its inverse. Otherwise, the good agreement between NMR and dielectric constant ${ }^{9,18}$ (which is natural because both techniques sample the molecular orientation) would get completely lost.

The TG experiment ${ }^{37}$ measures both the damping of longitudinal sound waves and a longer structural relaxation time. $^{47,48}$ One does not get the longitudinal sound wave relaxation time directly, but one can extract it from the temperature dependence of the damping. With a fitted $\beta=0.5$, the damping of the sound waves translates into the two

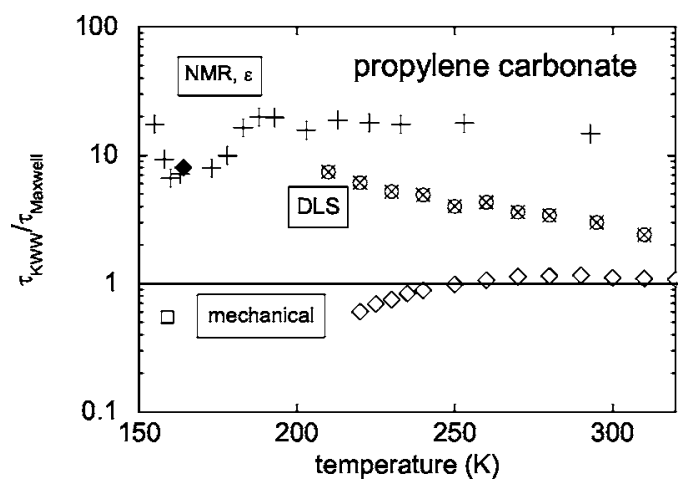

FIG. 3. Kohlrausch-Williams-Watts relaxation times in propylene carbonate, normalized to the Maxwell time as described in the text. Symbols: open square: shear (Ref. 13), open diamonds: longitudinal Brillouin (Ref. 14), pluses: dielectric (Ref. 15), full diamonds: NMR (Ref. 9), and open circles with cross: DLS (Ref. 55).

circles with crosses at 280 and $290 \mathrm{~K}$ in Fig. 2. In this case, one sees the splitting of time scales within experiments with a single sample and with the same temperature sensor.

In the structural relaxation of the transient grating experiment, the heat of the phonon bath transforms into structural potential energy, thereby expanding the sample. The relaxation time of this process is intimately related to the relaxation times of the heat capacity and of the density, which in turn are related to each other. The latter relation has been discussed earlier in several papers. ${ }^{49-53}$ Photon correlation spectroscopy (PCS) measures the refraction index fluctuations on the scale of the wavelength of the light, essentially density fluctuations. So it is not surprising to find the structural TG relaxation times close to those of PCS and heat capacity. The neutron spin-echo measurements at the first sharp diffraction peak sample the decay of the short range order of the molecular array. Again, it is not unexpected to find them close to those of the density and the entropy. What is surprising is to find the dielectric and the NMR times in the same group, because we are used to think of them as a single-molecule property ${ }^{8}$ and not as a collective process.

The idea of two different time scales (or an initial and final stage of the same process) is further supported by the different shape of the relaxation functions for the two groups of Fig. 2. The mechanical data have a decidedly larger stretching $\left(\beta_{\mathrm{KWw}} \approx 0.4-0.5\right)$ than the heat capacity, the dielectric constant, and the neutron spin-echo signal $\left(\beta_{\mathrm{KWW}}\right.$ $\approx 0.55-0.7$ ).

The same splitting of time scales, though not for so many different techniques as in the heavily studied case of glycerol, has been found for propylene carbonate ${ }^{14}$ and has been discussed in the framework of the mode-coupling theory. ${ }^{54}$ Note that this time scale splitting is not the twostage scenario of the mode-coupling theory, because both time scales move together with the Maxwell time. In fact, in Ref. 14 the $\alpha$ process of the theory was not attributed to the slower but to the faster process.

With the parameters in Table I, one can again relate the measured Kohlrausch relaxation times to the Maxwell time. As in glycerol, mechanical shear ${ }^{13}$ and Brillouin ${ }^{14}$ data in Fig. 3 show a decade faster decay than NMR (Ref. 9) and dielectric ${ }^{15}$ measurements, while DLS data ${ }^{55}$ lie in between. 


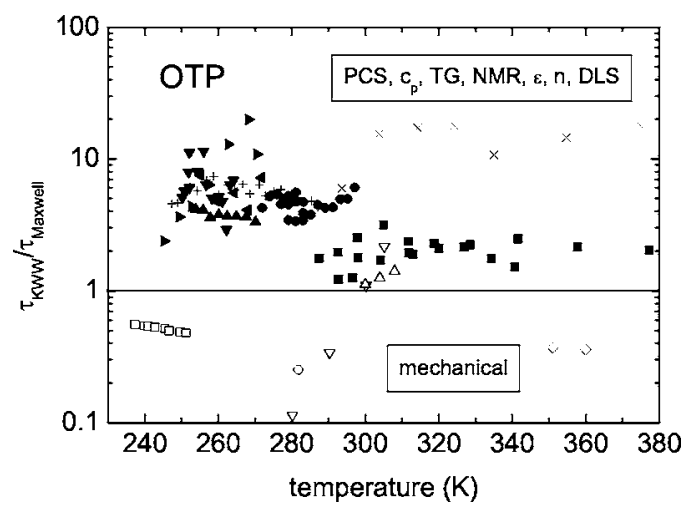

FIG. 4. Kohlrausch-Williams-Watts relaxation times in OTP, normalized to the Maxwell time as described in the text. Symbols: open squares: shear (Ref. 21), open circles: longitudinal acoustic (Ref. 31), open up triangles: TG (Ref. 56), open down triangles: transverse Brillouin (Ref. 30), open diamonds: longitudinal Brillouin (Ref. 31), pluses: dielectric (Ref. 61), full down triangles: NMR (Ref. 60), full up triangles: heat capacity (Ref. 57), full right triangles: PCS (Ref. 59), full left triangles: PCS (Ref. 58), full circles: TG (Ref. 56), full squares: neutron spin echo (Ref. 62), and crosses: DLS (Refs. 59 and 63).

Figure 4 shows again a heavily studied case, OTP. Mechanical measurements include a shear compliance study, ${ }^{21}$ longitudinal ultrasonic data, ${ }^{31}$ a transient grating experiment, ${ }^{56}$ a transverse Brillouin measurement, ${ }^{30}$ and a thorough analysis of longitudinal Brillouin light and $\mathrm{x}$-ray scatterings. ${ }^{31}$ Of these, the shear compliance and the ultrasonic and the longitudinal Brillouin data follow the Kohlrausch expectation of Eq. (5), but the transverse Brillouin data and the longitudinal sound wave part of the transient grating results do not; they show a sudden rise at about 290 $\mathrm{K}$. The reason for this deviation is clearly revealed in the analysis of Monaco et al. ${ }^{31}$ At $290 \mathrm{~K}$, the Johari-Goldstein peak merges with the main $\alpha$ relaxation. At such a point, our analysis in terms of a single Kohlrausch function is bound to fail.

Otherwise, Fig. 4 corroborates the results in Fig. 2. Again, the structural relaxation time from the $\mathrm{TG}$ experiment, ${ }^{56}$ heat capacity, ${ }^{57} \mathrm{PCS},{ }^{58,59}$ and NMR (Ref. 60) lie close to the dielectric data. ${ }^{61}$ The neutron data at the first sharp diffraction peak ${ }^{62}$ lie a bit lower but do still clearly belong to the upper group. The dynamic light scattering points $^{59,63}$ do not lie between the two groups as in glycerol and propylene carbonate but have higher relaxation times than all the other experiments.

In OTP, there is a rather convincing explanation of the NMR data in terms of a single-molecule picture, ${ }^{60}$ describing them in terms of rotational diffusion which follows the Debye-Stokes-Einstein equation

$$
D_{\text {trans }}=\frac{k_{B} T}{6 \pi \eta r_{H}}=\frac{4}{3} r_{H}^{2} D_{\mathrm{rot}},
$$

where $D_{\text {trans }}$ is the translational diffusion constant of the molecule and $D_{\text {rot }}$ is its rotational diffusion constant. For continuous rotational diffusion, the relaxation time for the Legendre polynomials is

$$
\tau_{L, \text { rot }}=\frac{1}{L(L+1) D_{\text {rot }}},
$$

where $L$ is the order of the Legendre polynomial. For the dielectric signal, $L=1$, but the NMR measurements referenced so far are all two-pulse sequences for deuterated molecules, where $L=2$. The OTP data ${ }^{60}$ are well described with a hydrodynamic radius $r_{H}$ of $22 \mathrm{~nm}$, close to the value $r_{H}$ $=23 \mathrm{~nm}$ found in NMR field gradient measurements ${ }^{60}$ at temperatures above $1.2 T_{g}$. The values are smaller than the expected van der Waals radius of $37 \mathrm{~nm}$ but are still not too far away from it.

If one lowers the temperature, the rotational relaxation time follows the temperature dependence of the viscosity, while the translational diffusion begins to deviate towards higher values. The same decoupling between translational and rotational motions has been found in photobleaching experiments $^{64}$ with guest molecules in OTP and has been taken as evidence for dynamical heterogeneity. In these experiments, one observes an increase of the relaxation times with increasing molecular diameter as expected, giving additional support to the single-molecule concept.

But the GDB extension of this single-molecule picture to describe the relation between $G(\omega)$ and $\epsilon(\omega)$, Eq. (2), does not work. At the glass temperature of OTP with $G_{\infty}$ $=1.6 \mathrm{GPa}$, one calculates a $c_{r}$ of 80 from Eq. (3). This implies that the peak in $\epsilon^{\prime \prime}(\omega)$ should be at a factor of 80 lower in frequency than the one in $G^{\prime \prime}(\omega)$, while the experiment shows only a factor of 10 . Again, this discrepancy has been noted before. ${ }^{8}$ In this case, one cannot blame the difference between external and internal electric fields, because the dipole moment of OTP is very small.

In some cases, one even finds the peak in $\epsilon^{\prime \prime}(\omega)$ rather close to the one in $G^{\prime \prime}(\omega)$. In decahydroisoquinoline (DHIQ) at $T_{g}$, they lie only a factor of 1.6 apart, ${ }^{16}$ instead of the factor of about 100 that one expects.

Also, the single-molecule picture fails to explain the striking coincidence between dielectric and NMR relaxation times on one hand and heat capacity, density, and short range order relaxation times on the other.

We will pursue an alternative explanation for the data in Figs. 2-4, namely, that the flow or $\alpha$ process begins at short times with a breakdown of the mechanical rigidity (the lower half of points in Figs. 2-4). A decade in time later, there seems to be a final process which equilibrates everything, the density, the entropy, and the short range order (the upper half of points in the three figures). This final process equilibrates also the molecular orientation, an order of magnitude earlier in time than expected on the basis of the Debye concept. In fact, a recent aging experiment ${ }^{65}$ shows that the dielectric relaxation time is indeed the final aging relaxation time also in a number of other molecular glass formers.

A possible way to understand such a process is to postulate a configurational potential energy which has a small fraction of shear energy, able to decay within the mechanical relaxation time, while the large rest is merely feeding the shear energy. In a physical picture, one divides the potential energy of a given configuration into a long range shear part 
and everything else. This "everything else" is supposed to be harmonic, decaying only via the shear energy channel.

This is similar to the physical mechanism of the Debye process, where the feeding energy is the energy of the electric dipole in the electric field. For the configurational energy, one replaces the electric dipole energy by the mechanical energy of an harmonic oscillator. As we will see, this change leads to a different equation; the two cases are similar but not identical.

To formulate the concept quantitatively, let us consider a mechanical model, a small spring $r$ in series with a frequency-dependent spring $g(\omega)=G(\omega) / G_{\infty}$. The compliance of the combination is the sum of the two compliances. Normalizing this sum, one gets

$$
\Phi(\omega)=\frac{1+r}{1+r / g(\omega)} .
$$

This is the Fourier transform of the decay function of the configurational energy according to the postulate above. $\Phi(\omega)$ is 1 in the high-frequency limit and zero in the lowfrequency limit; it is a modulus function.

We further postulate that the decay of the configuration involves a complete reorientation of the molecules, so it is mirrored in the dielectric signal. In the dielectric susceptibility, one expects to see $1-\Phi(\omega)$. Then

$$
\frac{\epsilon(\omega)-n^{2}}{\epsilon_{\mathrm{low}}-n^{2}}=\frac{1-g(\omega)}{1+g(\omega) / r} .
$$

This relation differs from the Gemant-DiMarzio-Bishop relation, Eq. (2), by the $1-g(\omega)$ in the numerator.

We used Eq. (13) to fit the dielectric data of glycerol ${ }^{1,11}$ and propylene carbonate. ${ }^{15} g(\omega)$ was obtained by first fitting the dynamical shear data ${ }^{10,13}$ at the glass transition in terms of a KWW function and then shifting this function to the required temperature using the shift factors of the Maxwell time. In glycerol, we also took the slight change of the Kohlrausch $\beta$ of the shear with temperature into account.

To get a good fit, it turned out to be necessary to leave $n$ as a free parameter and to allow for a slight difference of the shift factor (remember that the shear and dielectric data stem from different laboratories). These deviations, however, remained small and of the order of the differences between the fit values for the two different dielectric glycerol experiments. ${ }^{1,11}$ For the glycerol fit shown in Fig. 1(a), the shift factor difference corresponded to a factor of 0.7 in relaxation time. The $n$ value was 1.67 instead of 1.50. For the propylene carbonate fit in Fig. 1(b), the shift factor difference corresponded to a factor of 0.6 in relaxation time, and $n$ was 1.99 instead of 1.48. The shear energy fraction $r$ was 0.19 in both fits. At higher temperatures, $r$ tended to diminish more strongly in propylene carbonate than in glycerol.

In glycerol, the so-called "excess wing" at higher frequencies has been extensively discussed. ${ }^{66-68}$ From aging ${ }^{66,67}$ and pressure experiments, ${ }^{68}$ one forms the impression that the excess wing is an unresolved secondary relaxation or the Johari-Goldstein peak. Equation (13) does not add to this evidence but demonstrates that one deals with both a dielectric and a mechanical excess wing. In fact, once the parameters are known, one can use the equation to calculate the expected shear response from the dielectric data. It remains to be seen, however, how far one can trust an implicit assumption of Eq. (13), namely, that the elementary relaxation processes behind the breakdown of the shear modulus have the same ratio of the mechanical to the dielectric dipole moment over the whole frequency range.

To conclude, we propose an alternative to the Debye concept, because the Debye scheme is unable to account for the dielectric data in glycerol, propylene carbonate, and ortho-terphenyl. The alternative is based on the finding that the dielectric relaxation time in glycerol and OTP is close to the ones for the density, the entropy, and the short range order. The scheme provides a good fit for broadband dielectric spectra of glycerol and propylene carbonate.

We thank Peter Lunkenheimer and Ernst Rössler for supplying their dielectric data and for helpful discussions.

${ }^{1}$ U. Schneider, P. Lunkenheimer, R. Brand, and A. Loidl, J. Non-Cryst. Solids 235-237, 173 (1998).

${ }^{2}$ A. Gemant, Trans. Faraday Soc. 31, 1582 (1935).

${ }^{3}$ E. A. DiMarzio and M. Bishop, J. Chem. Phys. 60, 3802 (1974).

${ }^{4}$ C. J. F. Böttcher, Theory of Electric Polarization (Elsevier, Amsterdam, 1973).

${ }^{5}$ L. Onsager, J. Am. Chem. Soc. 58, 1486 (1936).

${ }^{6}$ E. Fatuzzo and P. R. Mason, Proc. Phys. Soc. London 90, 729 (1967).

${ }^{7}$ K. Niss and B. Jakobsen, M.S. thesis, University of Roskilde, Denmark, 2003. Our Eq. (4) follows directly from their Eq. (4.5.2).

${ }^{8}$ I. Chang and H. Sillescu, J. Chem. Phys. 101, 8794 (1997), and further references therein.

${ }^{9}$ F. Qi, K. U. Schug, S. Dupont, A. Döß, R. Böhmer, H. Sillescu, H. Kolshorn, and H. Zimmermann, J. Chem. Phys. 112, 9455 (2000).

${ }^{10}$ K. Schröter and E. Donth, J. Chem. Phys. 113, 9101 (2000).

${ }^{11}$ A. Kudlik, S. Benkhof, T. Blochowicz, C. Tschirwitz, and E. A. Rössler, J. Mol. Struct. 479, 201 (1999).

${ }^{12}$ L. Comez, D. Fioretto, F. Scarponi, and G. Monaco, J. Chem. Phys. 119, 6032 (2003).

${ }^{13}$ K. Schröter and E. Donth, J. Non-Cryst. Solids 307-310, 270 (2002).

${ }^{14}$ A. Brodin, M. Frank, S. Wiebel, G. Shen, J. Wuttke, and H. Z. Cummins, Phys. Rev. E 65, 051503 (2002).

${ }^{15}$ U. Schneider, P. Lunkenheimer, R. Brand, and A. Loidl, Phys. Rev. E 59, 6924 (1999).

${ }^{16}$ K. Niss, B. Jakobsen, and N. B. Olsen, J. Chem. Phys. 123, 234510 (2005); B. Jakobsen, K. Niss, and N. B. Olsen, ibid. 123, 234511 (2005).

${ }^{17}$ K. L. Ngai and R. W. Rendell, Phys. Rev. B 41, 754 (1990).

${ }^{18}$ T. Blochowicz, A. Kudlik, S. Benkhoff, J. Senker, and E. Rössler, J. Chem. Phys. 110, 12011 (1999).

${ }^{19}$ C. P. Lindsey and G. D. Patterson, J. Chem. Phys. 73, 3348 (1980).

${ }^{20}$ F. Alvarez, A. Alegria, and J. Colmenero, Phys. Rev. B 44, 7306 (1991).

${ }^{21}$ D. J. Plazek, C. A. Bero, and I.-C. Chay, J. Non-Cryst. Solids 172-174, 181 (1994).

${ }^{22}$ D. J. Ferry, Viscoelastic Properties of Polymers, 3rd ed. (Wiley, New York, 1980), Chap. 3, Eq. (54).

${ }^{23}$ A. Bondeau and J. Huck, J. Phys. (Paris) 46, 1717 (1985).

${ }^{24}$ R. J. Greet and D. Turnbull, J. Chem. Phys. 46, 1243 (1967).

${ }^{25}$ R. J. Greet and J. H. Magill, J. Phys. Chem. 71, 1746 (1967).

${ }^{26}$ W. T. Laughlin and D. R. Uhlmann, J. Phys. Chem. 76, 2317 (1972).

${ }^{27}$ M. Cukierman, J. W. Lane, and D. R. Uhlmann, J. Chem. Phys. 59, 3639 (1973).

${ }^{28}$ F. Scarponi, L. Comez, D. Fioretto, and L. Palmieri, Phys. Rev. B 70, 054203 (2004).

${ }^{29}$ M. Elmroth, L. Börjesson, and L. M. Torell, Phys. Rev. Lett. 68, 79 (1992).

${ }^{30}$ C. Dreyfus, A. Aouadi, J. Gapinski, M. Matos-Lopes, W. Steffen, A. Patkowski, and R. Pick, Phys. Rev. E 68, 011204 (2003).

${ }^{31}$ G. Monaco, D. Fioretto, L. Comez, and G. Ruocco, Phys. Rev. E 63, 061502 (2001).

${ }^{32}$ F. Stickel, Ph.D. thesis, University of Mainz, Germany, 1995.

${ }^{33}$ R. Piccirelli and T. A. Litovitz, J. Acoust. Soc. Am. 29, 1009 (1957). 
${ }^{34}$ Y. K. Jeong, S. R. Nagel, and S. Bhattacharya, Phys. Rev. A 34, 602 (1986).

${ }^{35}$ T. Christensen and N. B. Olsen, Phys. Rev. B 49, 15396 (1994).

${ }^{36}$ Y. K. Jeong, Phys. Rev. A 36, 766 (1987).

${ }^{37}$ D. M. Paolucci and K. A. Nelson, J. Chem. Phys. 112, 6725 (2000).

${ }^{38}$ N. O. Birge and S. R. Nagel, Phys. Rev. Lett. 54, 2674 (1985); N. O. Birge, Phys. Rev. B 34, 1631 (1986).

${ }^{39}$ J. Korus, Ph.D. thesis, University of Halle, Germany, 1997.

${ }^{40}$ S. A. Reinsberg, X. H. Qiu, M. Wilhelm, H. W. Spiess, and M. D. Ediger, J. Chem. Phys. 114, 7299 (2001).

${ }^{41}$ H. Dux and Th. Dorfmüller, Chem. Phys. 40, 219 (1979).

${ }^{42}$ J. Wuttke, W. Petry, and S. Pouget, J. Chem. Phys. 105, 5177 (1996).

${ }^{43}$ A. Brodin and E. A. Rössler, Eur. Phys. J. B 44, 3 (2005).

${ }^{44}$ R. Meister, C. J. Marhoeffer, R. Sciamanda, L. Cotter, and T. Litovitz, J. Appl. Phys. 31, 854 (1960).

${ }^{45}$ T. Christensen and N. B. Olsen, J. Non-Cryst. Solids 172-174, 362 (1994)

${ }^{46}$ One can, in fact, get a reasonable agreement between longitudinal ultrasonic relaxation times and dielectric ones by evaluating the ultrasonic data in terms of a compliance (Ref. 34). But then one does not know what to do with the shear data.

${ }^{47}$ Y. Yang and K. A. Nelson, J. Chem. Phys. 103, 7722 (1995).

${ }^{48}$ A. Taschin, R. Torre, M. Ricci, M. Sampoli, C. Dreyfus, and R. M. Pick, Europhys. Lett. 53, 407 (2001).

${ }^{49}$ W. Götze and A. Latz, J. Phys.: Condens. Matter 1, 4169 (1989).

${ }^{50}$ J. Jäckle, Physica A 162, 377 (1990).

${ }^{51}$ J. K. Nielsen and J. C. Dyre, Phys. Rev. B 54, 15754 (1996).

${ }^{52}$ T. Christensen and N. B. Olsen, Prog. Theor. Phys. Suppl. 126, 273 (1997).
${ }^{53}$ S. L. Simon and G. B. McKenna, J. Chem. Phys. 107, 8678 (1997).

${ }^{54}$ W. Götze and L. Sjögren, Rep. Prog. Phys. 55, 241 (1992).

${ }^{55}$ W. M. Du, G. Li, H. Z. Cummins, M. Fuchs, J. Toulouse, and L. A. Knauss, Phys. Rev. E 49, 2192 (1994).

${ }^{56}$ R. Torre, A. Taschin, and M. Sampoli, Phys. Rev. E 64, 061504 (2001).

${ }^{57}$ H. Leyser, A. Schulte, W. Doster, and W. Petry, Phys. Rev. E 51, 5899 (1995).

${ }^{58}$ G. Fytas, Th. Dorfmüller, and C. H. Wang, J. Phys. Chem. 87, 5045 (1983).

${ }^{59}$ W. Steffen, A. Patkowski, H. Gläser, G. Meier, and E. W. Fischer, Phys. Rev. B 49, 2992 (1994).

${ }^{60}$ I. Chang, F. Fujara, B. Geil, G. Heuberger, T. Mangel, and H. Sillescu, J. Non-Cryst. Solids 172-174, 248 (1994).

${ }^{61}$ C. Hansen, F. Stickel, R. Richert, and E. W. Fischer, J. Chem. Phys. 107, 1086 (1997).

${ }^{62}$ A. Tölle, Rep. Prog. Phys. 64, 1473 (2001).

${ }^{63}$ H. Z. Cummins, Y. H. Hwang, G. Li, W. M. Du, W. Losert, and G. Q. Shen, J. Non-Cryst. Solids 235-237, 254 (1998).

${ }^{64}$ M. Cicerone, F. R. Blackburn, and M. D. Ediger, J. Chem. Phys. 102, 471 (1995).

${ }^{65}$ P. Lunkenheimer, R. Wehn, U. Schneider, and A. Loidl, Phys. Rev. Lett. 95, $055702(2005)$.

${ }^{66}$ U. Schneider, R. Brand, P. Lunkenheimer, and A. Loidl, Phys. Rev. Lett. 84, 5560 (2000).

${ }^{67}$ K. L. Ngai, P. Lunkenheimer, C. Leon, U. Schneider, R. Brand, and A. Loidl, J. Chem. Phys. 115, 1405 (2001).

${ }^{68}$ K. L. Ngai and M. Paluch, J. Chem. Phys. 120, 857 (2004). 